ISSN 1855-3966 (printed edn.), ISSN 1855-3974 (electronic edn.)

ARS MATHEMATICA CONTEMPORANEA 18 (2020) 87-103

https://doi.org/10.26493/1855-3974.1860.a3d

(Also available at http://amc-journal.eu)

\title{
An equivalent formulation of the Fan-Raspaud Conjecture and related problems
}

\author{
Giuseppe Mazzuoccolo \\ Dipartimento di Informatica, Università di Verona, Strada Le Grazie 15, Verona, Italy \\ Jean Paul Zerafa \\ Dipartimento di Scienze Fisiche, Informatiche e Matematiche, \\ Università di Modena e Reggio Emilia, Via Campi 213/B, Modena, Italy
}

Received 20 November 2018, accepted 17 December 2019, published online 7 July 2020

\begin{abstract}
In 1994, it was conjectured by Fan and Raspaud that every simple bridgeless cubic graph has three perfect matchings whose intersection is empty. In this paper we answer a question recently proposed by Mkrtchyan and Vardanyan, by giving an equivalent formulation of the Fan-Raspaud Conjecture. We also study a possibly weaker conjecture originally proposed by the first author, which states that in every simple bridgeless cubic graph there exist two perfect matchings such that the complement of their union is a bipartite graph. Here, we show that this conjecture can be equivalently stated using a variant of Petersen-colourings, we prove it for graphs having oddness at most four and we give a natural extension to bridgeless cubic multigraphs and to certain cubic graphs having bridges.
\end{abstract}

Keywords: Cubic graph, perfect matching, oddness, Fan-Raspaud Conjecture, Berge-Fulkerson Conjecture, Petersen-colouring.

Math. Subj. Class. (2020): 05C15, 05C70

\section{Introduction and terminology}

Many interesting problems in graph theory are about the behaviour of perfect matchings in cubic graphs. One of the early classical results was made by Petersen [28] and states that every bridgeless cubic graph has at least one perfect matching. Some years ago, one of the most prominent conjectures in this area was completely solved by Esperet et al. in [5]: the conjecture, proposed by Lovász and Plummer in the 1970s, stated that the number

E-mail addresses: giuseppe.mazzuoccolo@univr.it (Giuseppe Mazzuoccolo), jeanpaul.zerafa@unimore.it (Jean Paul Zerafa) 
of perfect matchings in a bridgeless cubic graph grows exponentially with its order (see [20]). However, many others are still open, such as Conjecture 2.1 proposed independently by Berge and Fulkerson in the 1970s as well, and Conjecture 2.2 by Fan and Raspaud (see [10] and [7], respectively). These two conjectures are related to the behaviour of the union and intersection of sets of perfect matchings, and properties of this kind are already largely studied: see, amongst others, [1, 2, 15, 16, 17, 19, 22, 23, 25, 30, 31]. In this paper we prove that a seemingly stronger version of the Fan-Raspaud Conjecture is equivalent to the classical formulation (Theorem 3.3), and so to another interesting formulation proposed in [21] (see also [18]). In the second part of the paper (Section 4 and Section 5), we study a weaker conjecture proposed by the first author in [24]: we show how we can state it in terms of a variant of Petersen-colourings (Proposition 4.1) and we prove it for cubic graphs of oddness four (Theorem 5.4). Although all mentioned conjectures are about simple cubic graphs without bridges, we extend our study of the union of two perfect matchings to bridgeless cubic multigraphs and to particular cubic graphs having bridges (Section 6.1 and Section 6.2).

Graphs considered in the sequel, unless otherwise stated, are simple connected bridgeless cubic graphs and so do not contain loops and parallel edges. Graphs that may contain parallel edges will be referred to as multigraphs. For a graph $G$, let $V(G)$ and $E(G)$ be the set of vertices and the set of edges of $G$, respectively. A matching of $G$ is a subset of $E(G)$ such that any two of its edges do not share a common vertex. For an integer $k \geq 0$, a $k$-factor of $G$ is a spanning subgraph of $G$ (not necessarily connected) such that the degree of every vertex is $k$. The edge-set of a 1-factor is said to be a perfect matching. The least number of odd cycles amongst all 2-factors of $G$, denoted by $\omega(G)$, is called the oddness of $G$, and is clearly even for a cubic graph since $G$ has an even number of vertices. For $M \subseteq E(G)$, we denote the graph $G \backslash M$ by $\bar{M}$. In particular, when $M$ is a perfect matching of $G$, then $\bar{M}$ is a 2 -factor of $G$. In this case, following the terminology used for instance in [8], if $\bar{M}$ has $\omega(G)$ odd cycles, then $M$ is said to be a minimal perfect matching.

A cut in $G$ is any set $X \subseteq E(G)$ such that $\bar{X}$ has more components than $G$, and no proper subset of $X$ has this property, i.e. for any $X^{\prime} \subset X, \overline{X^{\prime}}$ does not have more components than $G$. The set of edges with precisely one end in $W \subseteq V(G)$ is denoted by $\partial_{G} W$, or just $\partial W$ when it is obvious to which graph we are referring. Moreover, a cut $X$ is said to be odd if there exists a subset $W$ of $V(G)$ having odd cardinality such that $X=\partial W$.

We next define some standard operations on graphs that will be useful in the sequel. Let $G_{1}$ and $G_{2}$ be two bridgeless graphs (not necessarily cubic), and let $e_{1}$ and $e_{2}$ be two edges such that $e_{1}=u_{1} v_{1} \in E\left(G_{1}\right)$ and $e_{2}=u_{2} v_{2} \in E\left(G_{2}\right)$. A 2-cut connection on $u_{1} v_{1}$ and $u_{2} v_{2}$ is a graph operation that consists of constructing the new graph

$$
\left[G_{1}-e_{1}\right] \cup\left[G_{2}-e_{2}\right] \cup\left\{u_{1} u_{2}, v_{1} v_{2}\right\},
$$

and denoted by $G_{1}\left(u_{1} v_{1}\right) * G_{2}\left(u_{2} v_{2}\right)$. It is clear that another possible graph obtained by a 2-cut connection on $e_{1}$ and $e_{2}$ is $G_{1}\left(u_{1} v_{1}\right) * G_{2}\left(v_{2} u_{2}\right)$. Clearly, the two graphs obtained are bridgeless, and, unless otherwise stated, if it is not important which of these two graphs is obtained, we use the notation $G_{1}\left(e_{1}\right) * G_{2}\left(e_{2}\right)$ and we say that it is a graph obtained by a 2 -cut connection on $e_{1}$ and $e_{2}$.

Now, let $G_{1}$ and $G_{2}$ be two bridgeless cubic graphs, $v_{1} \in V\left(G_{1}\right)$ and $v_{2} \in V\left(G_{2}\right)$ such that the vertices adjacent to $v_{1}$ are $x_{1}, y_{1}$ and $z_{1}$, and those adjacent to $v_{2}$ are $x_{2}, y_{2}$ and $z_{2}$. A 3-cut connection (sometimes also known as the star product, see for instance [11]) on $v_{1}$ 
and $v_{2}$ is a graph operation that consists of constructing the new graph

$$
\left[G_{1}-v_{1}\right] \cup\left[G_{2}-v_{2}\right] \cup\left\{x_{1} x_{2}, y_{1} y_{2}, z_{1} z_{2}\right\}
$$

and denoted by $G_{1}\left(x_{1} y_{1} z_{1}\right) * G_{2}\left(x_{2} y_{2} z_{2}\right)$. The 3 -edge-cut $\left\{x_{1} x_{2}, y_{1} y_{2}, z_{1} z_{2}\right\}$ is referred to as the principal 3-edge cut (see for instance [9]). As in the case of 2-cut connections, other graphs can be obtained by a 3 -cut connection on $v_{1}$ and $v_{2}$, and, unless otherwise stated, if it is not important how the adjacencies in the principal 3-edge cut look like, we use the notation $G_{1}\left(v_{1}\right) * G_{2}\left(v_{2}\right)$ and we say that it is a graph obtained by a 3 -cut connection on $v_{1}$ and $v_{2}$. It is clear that any resulting graph is also bridgeless and cubic.

\section{A list of relevant conjectures}

One of the aims of this paper is to study the behaviour of perfect matchings in cubic graphs, more specifically the union of two perfect matchings (see Section 4 and Section 5). We relate this to well-known conjectures stated here below, in particular: the Berge-Fulkerson Conjecture and the Fan-Raspaud Conjecture.

Conjecture 2.1 (Berge-Fulkerson [10]). Every bridgeless cubic graph G admits six perfect matchings $M_{1}, \ldots, M_{6}$ such that any edge of $G$ belongs to exactly two of them.

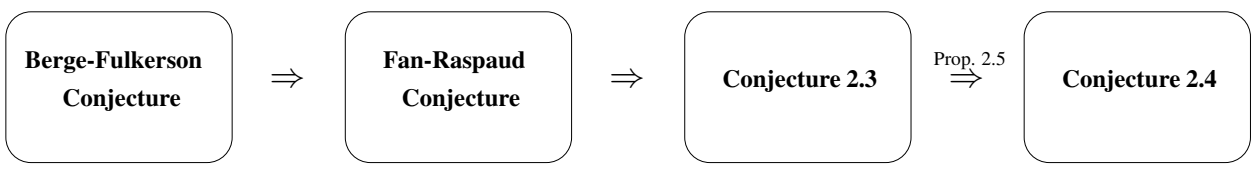

Figure 1: Conjectures mentioned and how they are related.

We also state here other (possibly weaker) conjectures implied by the above conjecture.

Conjecture 2.2 (Fan-Raspaud [7]). Every bridgeless cubic graph admits three perfect matchings $M_{1}, M_{2}$, and $M_{3}$ such that $M_{1} \cap M_{2} \cap M_{3}=\emptyset$.

In the sequel we will refer to three perfect matchings satisfying Conjecture 2.2 as an FR-triple. We can see that Conjecture 2.2 is immediately implied by the Berge-Fulkerson Conjecture, since we can take any three perfect matchings out of the six which satisfy Conjecture 2.1. A still weaker statement implied by the Fan-Raspaud Conjecture is the following:

Conjecture 2.3 ([21]). For each bridgeless cubic graph $G$, there exist two perfect matchings $M_{1}$ and $M_{2}$ such that $M_{1} \cap M_{2}$ contains no odd-cut of $G$.

We claim that any two perfect matchings out of the three in an FR-triple have no odd-cut in their intersection, in other words that Conjecture 2.2 implies Conjecture 2.3. For, suppose not. Then, without loss of generality, suppose that $M_{2} \cap M_{3}$ contains an odd-cut $X$. Hence, since every perfect matching has to intersect an odd-cut at least once, $\left|M_{1} \cap\left(M_{2} \cap M_{3}\right)\right| \geq\left|M_{1} \cap X\right| \geq 1$, a contradiction, since we assumed that $M_{1} \cap M_{2} \cap M_{3}$ is empty. In relation to the above, the first author proposed the following conjecture:

Conjecture 2.4 ( $S_{4}$-Conjecture [24]). For any bridgeless cubic graph $G$, there exist two perfect matchings such that the deletion of their union leaves a bipartite subgraph of $G$. 
For reasons which shall be obvious in Section 4 we let such a pair of perfect matchings be called an $S_{4}$-pair of $G$ and shall refer to Conjecture 2.4 as the $S_{4}$-Conjecture. We will first proceed by showing that this conjecture is implied by Conjecture 2.3 , and so, by what we have said so far, is a consequence of the Berge-Fulkerson Conjecture. In particular, we can see the $S_{4}$-Conjecture as Conjecture 2.3 restricted to odd-cuts $\partial V(C)$, where $C$ is an odd cycle of $G$.

Proposition 2.5. Conjecture 2.3 implies the $S_{4}$-Conjecture.

Proof. Let $M_{1}$ and $M_{2}$ be two perfect matchings such that their intersection does not contain any odd-cut. Consider $\overline{M_{1} \cup M_{2}}$, and suppose that it contains an odd cycle $C$. Then, all the edges of $\partial V(C)$ belong to $M_{1} \cap M_{2}$. If $\overline{\partial V(C)}$ has exactly two components, then $\partial V(C)$ is an odd-cut belonging to $M_{1} \cap M_{2}$, a contradiction. Therefore, $\overline{\partial V(C)}$ must have more than two components, say $k$, denoted by $C_{1}, C_{2}, \ldots, C_{k}$, where the first component $C_{1}$ is the cycle $C$. Let $\left[C_{1}, C_{j}\right]$ denote the set of edges between $C_{1}$ and $C_{j}$, for $j \in\{2, \ldots, k\}$. Since $\sum_{j=2}^{k}\left|\left[C_{1}, C_{j}\right]\right|=|\partial V(C)| \equiv 1(\bmod 2)$, there exists $j^{\prime} \in\{2, \ldots, k\}$, such that $\left|\left[C_{1}, C_{j^{\prime}}\right]\right| \equiv 1(\bmod 2)$. However, $\left[C_{1}, C_{j^{\prime}}\right]$ is an odd-cut which belongs to $M_{1} \cap M_{2}$, a contradiction.

\section{Statements equivalent to the Fan-Raspaud Conjecture}

Let $M_{1}, \ldots, M_{t}$ be a list of perfect matchings of $G$, and let $a \in E(G)$. We denote the number of times $a$ occurs in this list by $\nu_{G}\left[a: M_{1}, \ldots, M_{t}\right]$. When it is obvious which list of perfect matchings or which graph we are referring to, we will denote this as $\nu(a)$ and refer to it as the frequency of $a$. We will sometimes need to refer to the frequency of an ordered list of edges, say $(a, b, c)$, and we will do this by saying that the frequency of $(a, b, c)$ is $(i, j, k)$, for some integers $i, j$ and $k$. Mkrtchyan et al. [27] showed that the Fan-Raspaud Conjecture, i.e. Conjecture 2.2, is equivalent to the following:

Conjecture 3.1 ([27]). For each bridgeless cubic graph $G$, any edge $a \in E(G)$ and any $i \in$ $\{0,1,2\}$, there exist three perfect matchings $M_{1}, M_{2}$, and $M_{3}$ such that $M_{1} \cap M_{2} \cap M_{3}=\emptyset$ and $\nu_{G}\left[a: M_{1}, M_{2}, M_{3}\right]=i$.

In other words they show that if a graph has an FR-triple then, for every $i$ in $\{0,1,2\}$, there exists an FR-triple in which the frequency of a pre-chosen edge is exactly $i$. In the same paper, Mkrtchyan et al. state the following seemingly stronger version of the Fan-Raspaud Conjecture:

Conjecture 3.2 ([27]). Let $G$ be a bridgeless cubic graph, $w$ a vertex of $G$ and $i, j$ and $k$ three integers in $\{0,1,2\}$ such that $i+j+k=3$. Then, $G$ has an FR-triple in which the edges incident to $w$ in a given order have frequencies $(i, j, k)$.

This means that we can prescribe the frequencies to the three edges incident to a given vertex. At the end of [27], the authors remark that it would be interesting to show that Conjecture 3.2 is equivalent to the Fan-Raspaud Conjecture. We prove here that this is actually the case.

Theorem 3.3. Conjecture 3.2 is equivalent to the Fan-Raspaud Conjecture.

Proof. Since the Fan-Raspaud Conjecture is equivalent to Conjecture 3.1, it suffices to show the equivalence of Conjectures 3.1 and 3.2. The latter clearly implies the former, so 
assume Conjecture 3.1 is true and let $a, b$ and $c$ be the edges incident to $w$ such that the frequencies $(i, j, k)$ are to be assigned to $(a, b, c)$. It is sufficient to show that there exist two FR-triples in which the frequencies of $(a, b, c)$ are $(2,1,0)$ in one FR-triple (Case 1 below) and $(1,1,1)$ in the other FR-triple (Case 2 below).
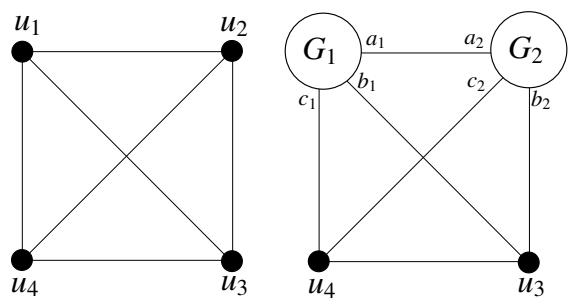

Figure 2: The graphs $K_{4}$ and $K_{4}^{*}$ in Case 1 of the proof of Theorem 3.3.

Case 1. Let $u_{1}, u_{2}, u_{3}$ and $u_{4}$ be the vertices of the complete graph $K_{4}$ as in Figure 2 . Consider two copies of $G$, and let the vertex $w$ in the $i^{\text {th }}$ copy of $G$ be denoted by $w_{i}$, for each $i \in\{1,2\}$. We apply a 3 -cut connection between $u_{i}$ and $w_{i}$, for each $i \in\{1,2\}$. With reference to this resulting graph, denoted by $K_{4}^{*}$, we refer to the copy of the graph $G-w$ at $u_{1}$ as $G_{1}$, and to the corresponding edges $a, b$ and $c$ as $a_{1}, b_{1}$ and $c_{1}$, respectively. The graph $G_{2}$ and the edges $a_{2}, b_{2}$ and $c_{2}$ are defined in a similar way, and the 3 -cut connection is done in such a way that $b_{1}$ and $b_{2}$ are adjacent, and also $c_{1}$ and $c_{2}$, as Figure 2 shows. Note also that $a_{1}$ and $a_{2}$ coincide in $K_{4}^{*}$. By our assumption, there exists an FR-triple $M_{1}, M_{2}$ and $M_{3}$ of $K_{4}^{*}$ in which the edge $u_{3} u_{4}$ has frequency 2. Without loss of generality, let $u_{3} u_{4} \in M_{1} \cap M_{2}$. Then, $a_{1}$ (and so $a_{2}$ ) must belong to $M_{1} \cap M_{2}$. Clearly, $a_{1}$ (and so $a_{2}$ ) cannot belong to $M_{3}$, and so the principal 3-edge-cuts with respect to $G_{1}$ and $G_{2}$ do not belong to $M_{3}$. If $b_{1} \in M_{3}$, then we are done, as then $M_{1}, M_{2}$ and $M_{3}$ restricted to $G_{1}$, together with $a$ and $b$ having the same frequencies as $a_{1}$ and $b_{1}$, induce an FR-triple of $G$ such that the frequencies of $(a, b, c)$ are $(2,1,0)$. So suppose $c_{1} \in M_{3}$. Then, $b_{2} \in M_{3}$, and so by a similar argument applied to $G_{2}$ and the corresponding edges, $M_{1}, M_{2}$ and $M_{3}$ induce an FR-triple in $G$ such that the frequencies of $(a, b, c)$ are $(2,1,0)$.
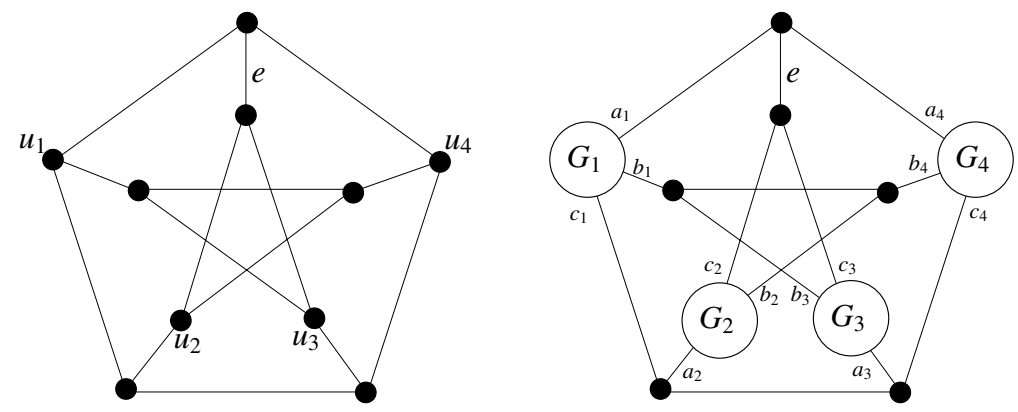

Figure 3: The graphs $P$ and $P^{*}$ in Case 2 of the proof of Theorem 3.3.

Case 2. Let $P$ be the Petersen graph and $\left\{u_{1}, u_{2}, u_{3}, u_{4}\right\}$ be a maximum independent set of vertices in $P$ as in Figure 3. Consider four copies of $G$. Let the vertex $w$ in the $i^{\text {th }}$ copy of $G$ be denoted by $w_{i}$, for each $i \in\{1, \ldots, 4\}$. Let $P^{*}$ be the graph obtained by applying 
a 3-cut connection between each $u_{i}$ and $w_{i}$, as shown in Figure 3. Similar to Case 1 we refer to the copy of $G-w$ at $u_{i}$ as $G_{i}$ and to the corresponding edges $a, b$ and $c$ as $a_{i}, b_{i}$ and $c_{i}$, respectively. Since we are assuming that Conjecture 3.1 is true, we can consider an FR-triple $M_{1}, M_{2}$ and $M_{3}$ of $P^{*}$ in which the edge $e$ incident to both $a_{1}$ and $a_{4}$ has frequency 2 . Without loss of generality, let the two perfect matchings containing $e$ be $M_{1}$ and $M_{2}$. The edges $a_{1}, c_{2}, c_{3}$ and $a_{4}$ are not contained in $M_{1}$ and neither $M_{2}$, since they are all incident to $e$, and so no principal 3-edge-cut leaving $G_{i}$ belongs to $M_{1}$ or $M_{2}$. Then, $M_{1}$ and $M_{2}$ induce perfect matchings of $P$ (clearly distinct), and since there are exactly two perfect matchings of $P$ containing $e$, we can assume that $M_{1}$ contains $\left\{e, b_{1}, a_{2}, a_{3}, b_{4}\right\}$, and $M_{2}$ contains $\left\{e, c_{1}, b_{2}, b_{3}, c_{4}\right\}$.

If the third perfect matching $M_{3}$ induces a perfect matching of the Petersen graph then the induced perfect matching cannot be one of the perfect matchings induced by $M_{1}$ and $M_{2}$ in $P$. Hence, since every two distinct perfect matchings of $P$ intersect in exactly one edge of $P$, there exists $i \in\{1,2,3,4\}$ such that the frequencies of $\left(a_{i}, b_{i}, c_{i}\right)$ are $(1,1,1)$ and so, $M_{1}, M_{2}$ and $M_{3}$ restricted to $G_{i}$, together with $a, b$ and $c$ having the same frequencies as $a_{i}, b_{i}$ and $c_{i}$, induce an FR-triple in $G$ with the needed property.

Therefore, suppose $M_{3}$ contains the principal 3-edge-cut of one of the $G_{i}$ s, say $G_{1}$ by symmetry of $P^{*}$. Thus, $a_{1}, b_{1}$ and $c_{1}$ belong to $M_{3}$. The perfect matching $M_{3}$ can intersect the principal 3-edge-cut at $G_{2}$ either in $b_{2}$ or $c_{2}$ (not both). If $c_{2} \in M_{3}$ we are done by the same reasoning above applied to $G_{2}$ and the corresponding edges. So suppose $b_{2} \in M_{2} \cap M_{3}$. Then, $c_{4} \in M_{3}$, and $M_{3}$ can only intersect the principal 3-edge-cut at $G_{3}$ in $c_{3}$, implying that the frequencies of $\left(a_{3}, b_{3}, c_{3}\right)$ are $(1,1,1)$ in $P^{*}$ and that $M_{1}, M_{2}$ and $M_{3}$ restricted to $G_{3}$, together with $a, b$ and $c$ having the same frequencies as $a_{3}, b_{3}$ and $c_{3}$, induce an FR-triple in $G$ with the needed property.

In [27] it is also shown that a minimal counterexample to Conjecture 3.2 is cyclically 4-edge-connected. It remains unknown whether a smallest counterexample to the original formulation of the Fan-Raspaud Conjecture has the same property. Indeed, we only prove that the two assertions are equivalent, but we cannot say whether a possible counterexample to Conjecture 3.2 is itself a counterexample to the original formulation.

\section{Statements equivalent to the $S_{4}$-Conjecture}

All conjectures presented in Section 2 are implied by a conjecture made by Jaeger in the late 1980s. In order to state it we need the following definitions. Let $G$ and $H$ be two graphs. An $H$-colouring of $G$ is a proper edge-colouring $f$ of $G$ with edges of $H$, such that for each vertex $u \in V(G)$, there exists a vertex $v \in V(H)$ with $f\left(\partial_{G}\{u\}\right) \subseteq \partial_{H}\{v\}$. If $G$ admits an $H$-colouring, then we will write $H \prec G$. In this paper we consider $S_{4}$-colourings of bridgeless cubic graphs, where $S_{4}$ is the multigraph shown in Figure 4.

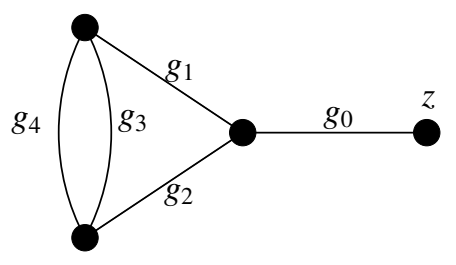

Figure 4: The multigraph $S_{4}$. 
The importance of $H$-colourings is mainly due to Jaeger's Conjecture [14] which states that each bridgeless cubic graph $G$ admits a $P$-colouring (where $P$ is again the Petersen graph). For recent results on $P$-colourings, known as Petersen-colourings, see for instance $[12,13,26,29]$. The following proposition shows why we choose to refer to a pair of perfect matchings whose deletion leaves a bipartite subgraph as an $S_{4}$-pair.

Proposition 4.1. Let $G$ be a bridgeless cubic graph, then $S_{4} \prec G$ if and only if $G$ has an $S_{4}$-pair.

Proof. Along the entire proof we denote the edges of $S_{4}$ by using the same labelling as in Figure 4. Let $M_{1}$ and $M_{2}$ be an $S_{4}$-pair of $G$. The graph induced by $M_{1} \cup M_{2}$, denoted by $G\left[M_{1} \cup M_{2}\right]$, is made up of even cycles and isolated edges, whilst the bipartite graph $\overline{M_{1} \cup M_{2}}$ is made up of even cycles and paths. We obtain an $S_{4}$-colouring of $G$ as follows:

- the isolated edges in $M_{1} \cup M_{2}$ are given colour $g_{0}$,

- the edges of the even cycles in $M_{1} \cup M_{2}$ are properly edge-coloured with $g_{3}$ and $g_{4}$, and

- the edges of the paths and even cycles in $\overline{M_{1} \cup M_{2}}$ are properly edge-coloured with $g_{1}$ and $g_{2}$.

One can clearly see that this gives an $S_{4}$-colouring of $G$. Conversely, assume that $S_{4} \prec G$. We are required to show that there exists an $S_{4}$-pair of $G$. Let $M_{1}$ be the set of edges of $G$ coloured $g_{3}$ and $g_{0}$, and let $M_{2}$ be the set of edges of $G$ coloured $g_{4}$ and $g_{0}$. If $e$ and $f$ are edges of $G$ coloured $g_{3}$ (or $g_{4}$ ) and $g_{0}$, respectively, then $e$ and $f$ cannot be adjacent, otherwise we contradict the $S_{4}$-colouring of $G$. Thus, $M_{1}$ and $M_{2}$ are matchings. Next, we show that they are in fact perfect matchings. This follows since for every vertex $v$ of $G, f\left(\partial_{G}\{v\}\right)$ is equal to $\left\{g_{1}, g_{3}, g_{4}\right\}$, or $\left\{g_{2}, g_{3}, g_{4}\right\}$, or $\left\{g_{0}, g_{1}, g_{2}\right\}$. Thus, $\overline{M_{1} \cup M_{2}}$ is the graph induced by the edges coloured $g_{1}$ and $g_{2}$, which clearly cannot induce an odd cycle.

Hence, by the previous proof, Conjecture 2.4 can be stated in terms of $S_{4}$-colourings, which clearly shows why we choose to refer to it as the $S_{4}$-Conjecture. In analogy to what we did for FR-triples, here we prove that for $S_{4}$-pairs we can prescribe the frequency of an edge and the frequencies of the edges leaving a vertex (the proof of the latter also implies that we can prescribe the frequencies of the edges of each 3-cut). Consider the following conjecture, analogous to Conjecture 3.1:

Conjecture 4.2. For any bridgeless cubic graph $G$, any edge $a \in E(G)$ and any $i \in$ $\{0,1,2\}$, there exists an $S_{4}$-pair, say $M_{1}$ and $M_{2}$, such that $\nu_{G}\left[a: M_{1}, M_{2}\right]=i$.

In Theorem 4.3 we show that the latter conjecture is actually equivalent to the $S_{4}$-Conjecture. The proof given in [27] to show the equivalence of the Fan-Raspaud Conjecture and Conjecture 3.1 is very similar to the proof we give here for the analogous case for the $S_{4}$-Conjecture, however, we need a slightly more complicated tool in our context.

Theorem 4.3. Conjecture 4.2 is equivalent to the $S_{4}$-Conjecture.

Proof. Clearly, Conjecture 4.2 implies the $S_{4}$-Conjecture so it suffices to show the converse. Assume the $S_{4}$-Conjecture to be true and let $f_{1}, f_{2}, f_{3}$ be three consecutive edges of $K_{4}$ inducing a path. Consider two copies of $G$. Let the edge $a$ in the $i^{\text {th }}$ copy of $G$ 
be denoted by $a_{i}$, for each $i \in\{1,2\}$. Let $K_{4}^{\prime}$ be the graph obtained by applying a 2 -cut connection between $f_{i}$ and $a_{i}$ for each $i \in\{1,2\}$. We refer to the copy of the graph $G-a$ on $f_{i}$ as $G_{i}$.

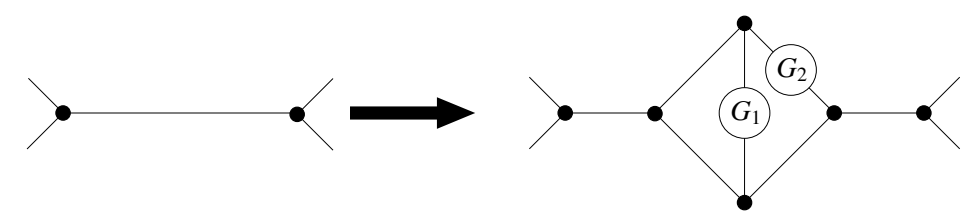

Figure 5: An edge in $P$ transformed into the corresponding structure in $H$.

Let $\left\{e_{1}, \ldots, e_{15}\right\}$ be the edges of the Petersen graph and let $T_{1}, \ldots, T_{15}$ be fifteen copies of $K_{4}^{\prime}$. For every $i \in\{1, \ldots, 15\}$, apply a 2-cut connection on $e_{i}$ and the edge $f_{3}$ of $T_{i}$. Consequently, every edge $e_{i}$ of the Petersen graph is transformed into the structure $E_{i}$ as in Figure 5, and we refer to $G_{1}$ and $G_{2}$ on $E_{i}$ as $G_{1}^{i}$ and $G_{2}^{i}$, respectively. Let $H$ be the resulting graph. By our assumption, there exists an $S_{4}$-pair of $H$, say $M_{1}$ and $M_{2}$, which induces a pair of two distinct perfect matchings in $P$, say $N_{1}$ and $N_{2}$, respectively. There exists an edge of $P$, say $e_{j}$, for some $j \in\{1, \ldots, 15\}$, such that $\nu_{P}\left[e_{j}: N_{1}, N_{2}\right]=1$, since every two distinct perfect matchings of $P$ have exactly one edge of $P$ in common. Hence, the restriction of $M_{1}$ and $M_{2}$ to the edge set of $G_{1}^{j}$, together with the edge $a$ having the same frequency as $e_{j}$, gives rise to an $S_{4}$-pair of $G$ in which the frequency of $a$ is 1 .

Moreover, there exists an edge of $P$, say $e_{k}$, for some $k \in\{1, \ldots, 15\}$, such that $\nu_{P}\left[e_{k}: N_{1}, N_{2}\right]=2$. Restricting $M_{1}$ and $M_{2}$ to the edge set of $G_{1}^{k}$, together with the edge $a$ having the same frequency as $e_{k}$, gives rise to an $S_{4}$-pair of $G$, in which the frequency of $a$ is 2 . Also, the restriction of $M_{1}$ and $M_{2}$ to the edge set of $G_{2}^{k}$ gives rise to an $S_{4}$-pair of $G\left(G_{2}^{k}\right.$ together with $\left.a\right)$, in which the frequency of $a$ is 0 , because if not, then there exists an odd cycle in $G$, say of length $\alpha$, passing through $a$ and having all its edges with frequency 0 . However, this would mean that there is an odd cycle of length $\alpha+4$ on $E_{k}$ in $\overline{M_{1} \cup M_{2}}$ (in $H$ ), a contradiction.

As in Section 3, we state an analogous conjecture to Conjecture 3.2, but for $S_{4}$-pairs:

Conjecture 4.4. Let $G$ be a bridgeless cubic graph, $w$ a vertex of $G$ and $i, j$ and $k$ three integers in $\{0,1,2\}$ such that $i+j+k=2$. Then, $G$ has an $S_{4}$-pair in which the edges incident to $w$ in a given order have frequencies $(i, j, k)$.

The following theorem shows that this conjecture is actually equivalent to Conjecture 4.2, and so to the $S_{4}$-Conjecture by Theorem 4.3 .

Theorem 4.5. Conjecture 4.4 is equivalent to the $S_{4}$-Conjecture.

Proof. Since the $S_{4}$-Conjecture is equivalent to Conjecture 4.2, it suffices to show the equivalence of Conjectures 4.2 and 4.4. Clearly, Conjecture 4.4 implies Conjecture 4.2 and so we only need to show the converse. Let $a, b$ and $c$ be the edges incident to $w$ such that the frequencies $(i, j, k)$ are to be assigned to $(a, b, c)$. We only need to prove the case when $(i, j, k)$ is equal to $(1,1,0)$, as all other cases follow from Conjecture 4.2.

Consider the graph $G(w) * P(v)$, where $P$ is the Petersen graph and $v$ is any vertex of $P$. We refer to the edges corresponding to $a, b$ and $c$ in $G(w) * P(v)$, as $a_{w}, b_{w}$ and $c_{w}$. 


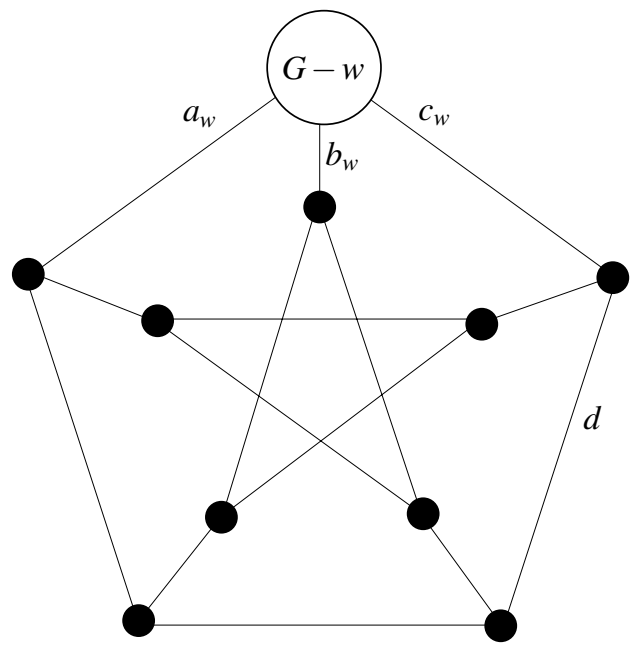

Figure 6: The graph $G(w) * P(v)$ from Theorem 4.5.

Let $d$ be an edge originally belonging to $P$ and adjacent to $c_{w}$ in $G(w) * P(v)$. Since we are assuming Conjecture 4.2 to be true, there exists an $S_{4}$-pair in $G(w) * P(v)$ in which $d$ has frequency 2 . If the frequencies of $\left(a_{w}, b_{w}, c_{w}\right)$ are $(1,1,0)$, then we are done, because the $S_{4}$-pair for $G(w) * P(v)$ restricted to the edges in $G-w$, together with $a$ and $b$ having the same frequencies as $a_{w}$ and $b_{w}$, give an $S_{4}$-pair for $G$ with the desired property. We claim that this must be the case. For, suppose not. Then, without loss of generality, the frequencies of $\left(a_{w}, b_{w}, c_{w}\right)$ are $(2,0,0)$. This implies that all the edges of $G(w) * P(v)$ originally in $P$ have either frequency 0 or 2 , since the two perfect matchings in the $S_{4}$-pair induce the same perfect matching in $P$. However, this implies that $P$ has a perfect matching whose complement is bipartite, a contradiction since $P$ is not 3 -edge-colourable.

As in [27], a minimal counterexample to Conjecture 4.4 (but not necessarily to the $S_{4}$-Conjecture) is cyclically 4 -edge-connected. We omit the proof of this result as it is very similar to the proof of Theorem 2 in [27].

\section{Further results on the $S_{4}$-Conjecture}

Little progress has been made on the Fan-Raspaud Conjecture so far. Bridgeless cubic graphs which trivially satisfy this conjecture are those which can be edge-covered by four perfect matchings. In this case, every three perfect matchings from a cover of this type form an FR-triple since every edge has frequency one or two with respect to this cover. Therefore, a possible counterexample to the Fan-Raspaud Conjecture should be searched for in the class of bridgeless cubic graphs whose edge-set cannot be covered by four perfect matchings, see for instance [6]. In 2009, Máčajová and Škoviera [22] shed some light on the Fan-Raspaud Conjecture by proving it for bridgeless cubic graphs having oddness two. One of the aims of this paper is to show that even if the $S_{4}$-Conjecture is still open, some results are easier to extend than the corresponding ones for the Fan-Raspaud Conjecture. Clearly, the result by Máčajová and Škoviera in [22] implies the following result: 
Theorem 5.1. Let $G$ be a bridgeless cubic graph of oddness two. Then, G has an $S_{4}$-pair.

We first give a proof of Theorem 5.1 in the same spirit of that used in [22], however much shorter since we are proving a weaker result.

Proof 1 of Theorem 5.1. Let $M_{1}$ be a minimal perfect matching of $G$, and let $C_{1}$ and $C_{2}$ be the two odd cycles in $\overline{M_{1}}$. Colour the even cycles in $\overline{M_{1}}$ using two colours, say 1 and 2. For each $i \in\{1,2\}$, let $E_{i}$ be the set of edges belonging to the even cycles in $\overline{M_{1}}$ and having colour $i$. In $G$, there must exist a path $Q$ whose edges alternate in $M_{1}$ and $E_{1}$ and whose end-vertices belong to $C_{1}$ and $C_{2}$, respectively, since $C_{1}$ and $C_{2}$ are odd cycles. Note that since the edges of $C_{1}$ and $C_{2}$ are not edges in $M_{1} \cup E_{1}$, every other vertex on $Q$ which is not an end-vertex does not belong to $C_{1}$ and $C_{2}$.

For each $i \in\{1,2\}$, let $v_{i}$ be the end-vertex of $Q$ belonging to $C_{i}$, and let $M_{C_{i}}$ be the unique perfect matching of $C_{i}-v_{i}$. Let $M_{2}:=\left(M_{1} \cap Q\right) \cup\left(E_{1} \backslash Q\right) \cup M_{C_{1}} \cup M_{C_{2}}$. Clearly, $M_{2}$ is a perfect matching of $G$ which intersects $C_{1}$ and $C_{2}$, and so $\overline{M_{1} \cup M_{2}}$ is bipartite.

We now give a second alternative proof of the same theorem using fractional perfect matchings, which we will show to be easier to use for graphs having larger oddness. Let $w$ be a vector in $\mathbb{R}^{|E(G)|}$. The entry of $w$ corresponding to $e \in E(G)$ is denoted by $w(e)$, and for $A \subseteq E(G)$, we let the weight of $A$, denoted by $w(A)$, to be equal to $\sum_{e \in A} w(e)$. The vector $w$ is said to be a fractional perfect matching of $G$ if:

1. $w(e) \in[0,1]$ for each $e \in E(G)$,

2. $w(\partial\{v\})=1$ for each $v \in V(G)$, and

3. $w(\partial W) \geq 1$ for each $W \subseteq V(G)$ of odd cardinality.

The following lemma is presented in [16] and it is a consequence of Edmonds' characterisation of perfect matching polytopes in [3].

Lemma 5.2. If $w$ is a fractional perfect matching in a graph $G$, and $c \in \mathbb{R}^{|E(G)|}$, then $G$ has a perfect matching $N$ such that

$$
c \cdot \chi^{N} \geq c \cdot w
$$

where $\cdot$ denotes the inner product. Moreover, there exists a perfect matching satisfying the above inequality and which contains exactly one edge of each odd-cut $X$ with $w(X)=1$.

Remark 5.3. If we let $w(e)=1 / 3$ for all $e \in E(G)$, for some graph $G$, then we know that $w$ is a fractional perfect matching of $G$. Also, since the weight of every 3 -cut is one, then by Lemma 5.2 there exists a perfect matching of $G$ containing exactly one edge of each 3-cut of $G$.

Proof 2 of Theorem 5.1. Let $M_{1}$ be a minimal perfect matching of $G$, and let $C_{1}$ and $C_{2}$ be the two odd cycles in $\overline{M_{1}}$. For each $i \in\{1,2\}$, let $e_{1}^{i}$ and $e_{2}^{i}$ be two adjacent edges belonging to $C_{i}$. We define the vector $c \in \mathbb{R}^{|E(G)|}$ such that

$$
c(e)= \begin{cases}1 & \text { if } e \in \cup_{i=1}^{2}\left\{e_{1}^{i}, e_{2}^{i}\right\} \\ 0 & \text { otherwise. }\end{cases}
$$


By Remark 5.3, we also know that if we let $w(e)=1 / 3$ for all $e \in E(G)$, then $w$ is a fractional perfect matching of $G$. Hence, by Lemma 5.2, there exists a perfect matching $M_{2}$ such that $c \cdot \chi^{M_{2}} \geq c \cdot w$, which implies that

$$
\left|\cup_{i=1}^{2}\left\{e_{1}^{i}, e_{2}^{i}\right\} \cap M_{2}\right| \geq 1 / 3 \times 2 \times 2=4 / 3>1 .
$$

Therefore, for each $i \in\{1,2\}$, there exists exactly one $j \in\{1,2\}$ such that $e_{j}^{i} \in M_{2}$. Hence, $M_{2}$ intersects $C_{1}$ and $C_{2}$ and so $\overline{M_{1} \cup M_{2}}$ is bipartite.

Using the same idea as in Proof 2 of Theorem 5.1, we also prove that the $S_{4}$-Conjecture is true for graphs having oddness four.

Theorem 5.4. Let $G$ be a bridgeless cubic graph of oddness four. Then, $G$ has an $S_{4}$-pair.

Proof. Let $M_{1}$ be a minimal perfect matching of $G$, and let $C_{1}, C_{2}, C_{3}$ and $C_{4}$ be the four odd cycles in $\overline{M_{1}}$. By Remark 5.3, there exists a perfect matching $N$ of $G$ such that if $G$ has any 3 -cuts, then $N$ intersects every 3 -cut of $G$ in one edge. Moreover, for every $i \in\{1, \ldots, 4\}$, there exists at least a pair of adjacent edges $e_{1}^{i}$ and $e_{2}^{i}$ belonging to $C_{i} \cap \bar{N}$. We define the vector $c \in \mathbb{R}^{|E(G)|}$ such that

$$
c(e)= \begin{cases}1 & \text { if } e \in \cup_{i=1}^{4}\left\{e_{1}^{i}, e_{2}^{i}\right\} \\ 0 & \text { otherwise. }\end{cases}
$$

We also define the vector $w \in \mathbb{R}^{|E(G)|}$ as follows:

$$
w(e)= \begin{cases}1 / 5 & \text { if } e \in N, \\ 2 / 5 & \text { otherwise }\end{cases}
$$

The vector $w$ is clearly a fractional perfect matching of $G$ because, in particular, $N$ intersects every 3 -cut in one edge and so $w(X) \geq 1$ for each odd-cut $X$ of $G$. Hence, by Lemma 5.2, there exists a perfect matching $M_{2}$ such that $c \cdot \chi^{M_{2}} \geq c \cdot w$, which implies that

$$
\left|\cup_{i=1}^{4}\left\{e_{1}^{i}, e_{2}^{i}\right\} \cap M_{2}\right| \geq 2 / 5 \times 2 \times 4=16 / 5>3 .
$$

Therefore, for each $i \in\{1, \ldots, 4\}$, there exists exactly one $j \in\{1,2\}$ such that $e_{j}^{i} \in M_{2}$. Hence, $M_{2}$ intersects $C_{1}, C_{2}, C_{3}$ and $C_{4}$ and so $\overline{M_{1} \cup M_{2}}$ is bipartite.

As the above proofs show us, extending results with respect to the $S_{4}$-Conjecture is easier than in the case of the Fan-Raspaud Conjecture and this is why we believe that a proof of the $S_{4}$-conjecture could be a first feasible step towards a solution of the Fan-Raspaud Conjecture. For graphs having oddness at least six we are not able to prove the existence of an $S_{4}$-pair and we wonder how many perfect matchings we need such that the complement of their union is bipartite. In the next proposition we use the technique used in Theorem 5.4 and show that given a bridgeless cubic graph $G$, if $\omega(G) \leq 5^{k-1}-1$ for some positive integer $k$, then there exist $k$ perfect matchings such that the complement of their union is bipartite. Note that for $k=2$ we obtain $\omega(G) \leq 4$.

Proposition 5.5. Let $G$ be a bridgeless cubic graph and let $\mathcal{C}$ be a collection of disjoint odd cycles in $G$ such that $|\mathcal{C}| \leq 5^{k-1}-1$ for some positive integer $k$. Then, there exist $k-1$ perfect matchings of $G$, say $M_{1}, \ldots, M_{k-1}$, such that for every $C \in \mathcal{C}$, there exists $j \in\{1, \ldots, k-1\}$ for which $C \cap M_{j} \neq \emptyset$. Moreover, if $\omega(G) \leq 5^{k-1}-1$, then there exist $k$ perfect matchings such that the complement of their union is bipartite. 
Proof. We proceed by induction on $k$. For $k=1$, the assertion trivially holds since $\mathcal{C}$ is the empty set. Assume the result is true for some $k \geq 1$ and consider $k+1$. Let $C_{1}, C_{2}, \ldots, C_{t}$, with $t \leq 5^{k}-1$, be the odd cycles of $G$ in $\mathcal{C}$. Let $N$ be a perfect matching of $G$ which intersects every 3 -cut of $G$ once. For every $i \in\{1, \ldots, t\}$, there exists at least a pair of adjacent edges $e_{1}^{i}$ and $e_{2}^{i}$ belonging to $C_{i} \cap \bar{N}$. We define the vector $c \in \mathbb{R}^{|E(G)|}$ such that

$$
c(e)= \begin{cases}1 & \text { if } e \in \cup_{i=1}^{t}\left\{e_{1}^{i}, e_{2}^{i}\right\}, \\ 0 & \text { otherwise. }\end{cases}
$$

We also define the vector $w \in \mathbb{R}^{|E(G)|}$ as follows:

$$
w(e)= \begin{cases}1 / 5 & \text { if } e \in N, \\ 2 / 5 & \text { otherwise }\end{cases}
$$

As in the proof of Theorem 5.4, $w$ is a fractional perfect matching of $G$ and by Lemma 5.2 there exists a perfect matching $M_{k}$ such that $c \cdot \chi^{M_{k}} \geq c \cdot w$. This implies that

$$
\left|\cup_{i=1}^{t}\left\{e_{1}^{i}, e_{2}^{i}\right\} \cap M_{k}\right| \geq 2 \times 2 / 5 \times t .
$$

Let $\mathcal{C}^{\prime}$ be the subset of $\mathcal{C}$ which contains the odd cycles of $\mathcal{C}$ with no edge of $M_{k}$. Then, $\left|\mathcal{C}^{\prime}\right| \leq|\mathcal{C}|-\frac{4}{5} t=t-\frac{4}{5} t=\frac{t}{5} \leq 5^{k-1}-\frac{1}{5}$, and so $\left|\mathcal{C}^{\prime}\right| \leq 5^{k-1}-1$. By induction, there exist $k-1$ perfect matchings of $G$, say $M_{1}, \ldots, M_{k-1}$, having the required property with respect to $\mathcal{C}^{\prime}$. Therefore, $M_{1}, \ldots, M_{k}$ intersect all odd cycles in $\mathcal{C}$. The second part of the statement easily follows by considering $\mathcal{C}$ to be the set of odd cycles in the complement of a minimal perfect matching $M$ of $G$, since the union of $M$ with the $k-1$ perfect matchings which intersect all the odd cycles in $\mathcal{C}$ has a bipartite complement.

Remark 5.6. We note that with every step made in the proof of Proposition 5.5, one could update the weight $w$ of the edges using the methods presented in $[16,23]$ which gives a slightly better upper bound for $\omega(G)$. For reasons of simplicity and brevity, we prefer the present weaker version of Proposition 5.5.

\section{Extension of the $S_{4}$-Conjecture to larger classes of cubic graphs}

\subsection{Multigraphs}

In this section we discuss natural extensions of some previous conjectures to bridgeless cubic multigraphs. We note that bridgeless cubic multigraphs cannot contain any loops. We will make use of the following standard operation on parallel edges, referred to as smoothing. Let $G^{\prime}$ be a bridgeless cubic multigraph. Let $u$ and $v$ be two vertices in $G^{\prime}$ such that there are exactly two parallel edges between them.

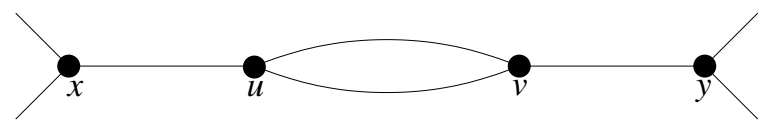

Figure 7: Vertices $x, u, v$ and $y$ in $G^{\prime}$.

Let $x$ and $y$ be the vertices adjacent to $u$ and $v$, respectively (see Figure 7). We say that we smooth $u v$ if we delete the vertices $u$ and $v$ from $G^{\prime}$ and add an edge between $x$ 
and $y$ (even if $x$ and $y$ are already adjacent in $G^{\prime}$ ). One can easily see that the resulting multigraph, say $G$, after smoothing $u v$ is again bridgeless and cubic.

In what follows, we will say that a perfect matching $M$ of $G$ and a perfect matching $M^{\prime}$ of $G^{\prime}$ are corresponding perfect matchings if the following holds:

$$
M= \begin{cases}M^{\prime} \cup x y-\{x u, v y\} & \text { if } x u \in M^{\prime} \\ M^{\prime}-u v & \text { otherwise. }\end{cases}
$$

The following theorem can be easily proved by using smoothing operations.

Theorem 6.1. The $S_{4}$-Conjecture is true if and only if every bridgeless cubic multigraph has an $S_{4}$-pair.

Now we show that Conjecture 4.4 can also be extended to multigraphs.

Theorem 6.2. Let $i, j$ and $k$ be three integers in $\{0,1,2\}$ such that $i+j+k=2$ and let $w$ be a vertex in a bridgeless cubic multigraph $G^{\prime}$. Then, the $S_{4}$-Conjecture is true if and only if $G^{\prime}$ has an $S_{4}$-pair in which the edges incident to $w$ in a given order have frequencies $(i, j, k)$.

Proof. It suffices to assume that the $S_{4}$-Conjecture is true and only show the forward direction, by Theorem 6.1. Let $G^{\prime}$ be a minimal counterexample and suppose it has some parallel edges. If $G^{\prime}=C_{2,3}$ then the result clearly follows. So assume $G^{\prime} \neq C_{2,3}$. Let $a, b$ and $c$ be the edges incident to $w$ such that the frequencies $(i, j, k)$ are to be assigned to $(a, b, c)$. We proceed by considering two cases: when $w$ has two parallel edges incident to it (Figure 8) and otherwise (Figure 9).

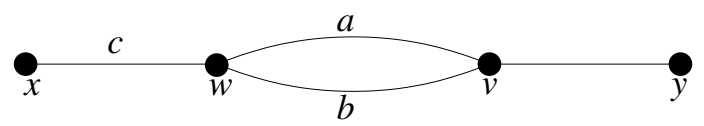

Figure 8: Case 1 in the proof of Theorem 6.2.

Case 1. Let $G$ be the resulting multigraph after smoothing $w v$. By minimality of $G^{\prime}, G$ has an $S_{4}$-pair (say $M_{1}$ and $M_{2}$ ) in which $\nu(x y)=k$. It is easy to see that a pair of corresponding perfect matchings in $G^{\prime}$ give $\nu_{G^{\prime}}(c)=\nu_{G^{\prime}}(v y)=k$ and can be chosen in such a way such that $\nu_{G^{\prime}}(a)=i$ and $\nu_{G^{\prime}}(b)=j$, a contradiction to our initial assumption. Therefore, we must have Case 2.

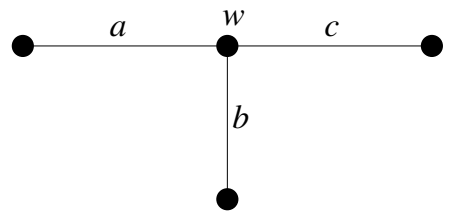

Figure 9: Case 2 in the proof of Theorem 6.2.

Case 2. Let $G$ be the resulting multigraph after smoothing some parallel edge in $G^{\prime}$ and let $a_{w}, b_{w}$ and $c_{w}$ be the corresponding edges incident to $w$ in $G$ after smoothing is done. 
In $G$, there exists an $S_{4}$-pair such that the frequencies of $\left(a_{w}, b_{w}, c_{w}\right)$ are equal to $(i, j, k)$. Clearly, the corresponding perfect matchings in $G^{\prime}$ form an $S_{4}$-pair in which the frequencies of $(a, b, c)$ are $(i, j, k)$, a contradiction, proving Theorem 6.2.

Using the same ideas as in Theorem 6.1 and Theorem 6.2 one can also state analogous results for the Fan-Raspaud Conjecture in terms of multigraphs.

\subsection{Graphs having bridges}

Since every perfect matching must intersect every bridge of a cubic graph, then the FanRaspaud Conjecture cannot be extended to cubic graphs containing bridges. The situation is quite different for the $S_{4}$-Conjecture as Theorem 6.3 shows. By Errera's Theorem [4] we know that if all the bridges of a connected cubic graph lie on a single path, then the graph has a perfect matching. We use this idea to show that there can be graphs with bridges that can have an $S_{4}$-pair.

Theorem 6.3. Let $G$ be a connected cubic graph having $k$ bridges, all of which lie on a single path, for some positive integer $k$. If the $S_{4}$-Conjecture is true, then $G$ admits an $S_{4}$-pair.

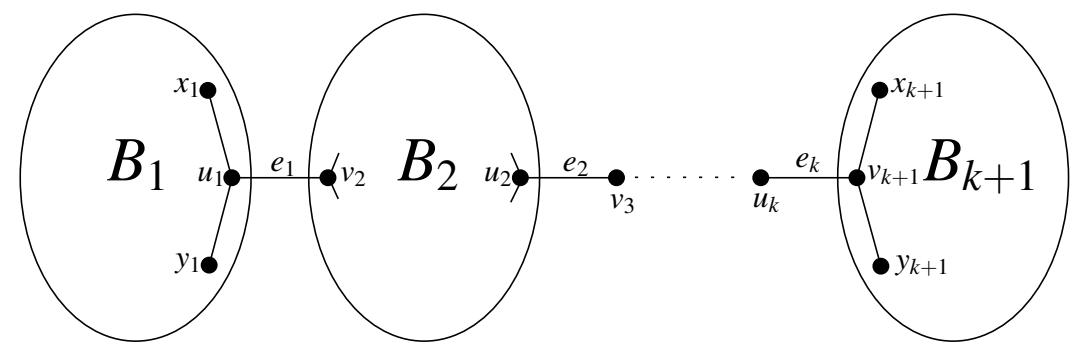

Figure 10: $G$ with $k$ bridges lying all on the same path.

Proof. Let $B_{1}, B_{2}, \ldots, B_{k+1}$ be the 2-connected components of $G$ and let $e_{1}, \ldots, e_{k}$ be the bridges of $G$ such that $e_{i}=u_{i} v_{i+1}$ for each $i \in\{1, \ldots, k\}$, where $u_{i} \in V\left(B_{i}\right)$ and $v_{i+1} \in V\left(B_{i+1}\right)$. Let $x_{1}$ and $y_{1}$ be the two vertices adjacent to $u_{1}$ in $B_{1}$, and let $x_{k+1}$ and $y_{k+1}$ be the two vertices adjacent to $v_{k+1}$ in $B_{k+1}$. Let $B_{1}^{\prime}=\left(B_{1}-u_{1}\right) \cup x_{1} y_{1}$ and $B_{k+1}^{\prime}=\left(B_{k+1}-v_{k+1}\right) \cup x_{k+1} y_{k+1}$. Also, let $B_{i}^{\prime}=B_{i} \cup v_{i} u_{i}$ for every $i \in\{2, \ldots, k\}$. Clearly, $B_{1}^{\prime}, \ldots, B_{k+1}^{\prime}$ are bridgeless cubic multigraphs. Since we are assuming that the $S_{4}$-Conjecture holds, then, by Theorem 6.1 , for every $i \in\{1, \ldots, k+1\}, B_{i}^{\prime}$ has an $S_{4}$-pair, say $M_{1}^{i}$ and $M_{2}^{i}$. Using Theorem 6.2, we choose the $S_{4}$-pair in:

- $B_{1}^{\prime}$, such that the two edges originally incident to $x_{1}$ (not $x_{1} u_{1}$ ) both have frequency 1 ,

- $B_{i}^{\prime}$, for each $i \in\{2, \ldots, k\}$, such that $\nu_{B_{i}^{\prime}}\left(v_{i} u_{i}\right)=2$, and

- $B_{k+1}^{\prime}$, such that the two edges originally incident to $x_{k+1}$ (not $x_{k+1} v_{k+1}$ ) both have frequency 1 .

Let $M_{1}:=\left(\cup_{i=1}^{k+1} M_{1}^{i}\right) \cup\left(\cup_{j=1}^{k} e_{j}\right)-\cup_{l=2}^{k} v_{l} u_{l}$, and let $M_{2}:=\left(\cup_{i=1}^{k+1} M_{2}^{i}\right) \cup\left(\cup_{j=1}^{k} e_{j}\right)-$ $\cup_{l=2}^{k} v_{l} u_{l}$. Then, $M_{1}$ and $M_{2}$ are an $S_{4}$-pair of $G$. 
Finally, we remark that there exist cubic graphs which admit a perfect matching however do not have an $S_{4}$-pair. For example, since the edges $u_{i} v_{i}$ in Figure 11 are bridges, then they must be in any perfect matching. Consequently, every pair of perfect matchings do not intersect the edges of the odd cycle $T$. This shows that it is not possible to extend the $S_{4}$-Conjecture to the entire class of cubic graphs.

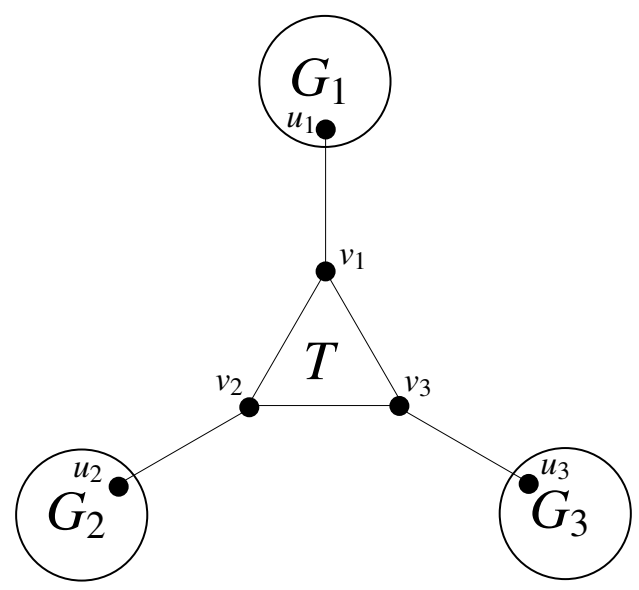

Figure 11: A cubic graph with bridges having no $S_{4}$-pair.

\section{Remarks and problems}

Many problems about the topics presented above remain unsolved: apart from asking if we can solve the Fan-Raspaud Conjecture and the $S_{4}$-Conjecture completely, or at least partially for higher oddness, we do not know which are those graphs containing bridges which admit an $S_{4}$-pair and we do not know either if the $S_{4}$-Conjecture is equivalent to Conjecture 2.3. Here we would like to add some other specific open problems.

For a positive integer $k$, we define $\omega_{k}$ to be the largest integer such that any graph with oddness at most $\omega_{k}$, admits $k$ perfect matchings with a bipartite complement. Clearly, for $k=1$, we have $\omega_{1}=0$, since the existence of a perfect matching of $G$ with a bipartite complement is equivalent to the 3 -edge-colourability of $G$. Moreover, the $S_{4}$-Conjecture is equivalent to $\omega_{k}=\infty$, for $k \geq 2$, but a complete result to this is still elusive. Proposition 5.5 (see also Remark 5.6) gives a lower bound for $\omega_{k}$ and it would be interesting if this lower bound can be significantly improved. We believe that the following problem, weaker than the $S_{4}$-Conjecture, is another possible step forward.

Problem 7.1. Prove the existence of a constant $k$ such that every bridgeless cubic graph admits $k$ perfect matchings whose union has a bipartite complement.

It is also known that not every perfect matching can be extended to an FR-triple and neither to a Berge-Fulkerson cover, where the latter is a collection of six perfect matchings which cover the edge set exactly twice. We do not see a way how to produce a similar argument for $S_{4}$-pairs and so we also suggest the following problem.

Problem 7.2. Establish whether any perfect matching of a bridgeless cubic graph can be extended to an $S_{4}$-pair. 
It can be shown that Problem 7.2 is equivalent to saying that given any collection of disjoint odd cycles in a bridgeless cubic graph, then there exists a perfect matching which intersects all the odd cycles in this collection.

\section{ORCID iDs}

Giuseppe Mazzuoccolo (D) https://orcid.org/0000-0001-7775-065X

Jean Paul Zerafa iD https://orcid.org/0000-0002-3159-2980

\section{References}

[1] M. Abreu, T. Kaiser, D. Labbate and G. Mazzuoccolo, Treelike snarks, Electron. J. Combin. 23 (2016), \#P3.54, doi:10.37236/6008.

[2] A. Bonisoli and D. Cariolaro, Excessive factorizations of regular graphs, in: A. Bondy, J. Fonlupt, J.-L. Fouquet, J.-C. Fournier and J. L. Ramírez Alfonsín (eds.), Graph Theory in Paris, Springer, Trends in Mathematics, pp. 73-84, 2007, doi:10.1007/978-3-7643-7400-6_7, proceedings of the conference (GTO4) in memory of Claude Berge held in Paris, July 2004.

[3] J. Edmonds, Maximum matching and a polyhedron with 0,1-vertices, J. Res. Nat. Bur. Standards 69B (1965), 125-130, doi:10.6028/jres.069B.013.

[4] A. Errera, Une démonstration du théorème de Petersen, Mathesis 36 (1922), 56-61.

[5] L. Esperet, F. Kardoš, A. D. King, D. Král and S. Norine, Exponentially many perfect matchings in cubic graphs, Adv. Math. 227 (2011), 1646-1664, doi:10.1016/j.aim.2011.03.015.

[6] L. Esperet and G. Mazzuoccolo, On cubic bridgeless graphs whose edge-set cannot be covered by four perfect matchings, J. Graph Theory 77 (2014), 144-157, doi:10.1002/jgt.21778.

[7] G. Fan and A. Raspaud, Fulkerson's conjecture and circuit covers, J. Comb. Theory Ser. B 61 (1994), 133-138, doi:10.1006/jctb.1994.1039.

[8] M. A. Fiol, G. Mazzuoccolo and E. Steffen, Measures of edge-uncolorability of cubic graphs, Electron. J. Combin. 25 (2018), \#P4.54, doi:10.37236/6848.

[9] J.-L. Fouquet and J.-M. Vanherpe, On the perfect matching index of bridgeless cubic graphs, 2009, preprint, arXiv:0904.1296 [CS.DM] .

[10] D. R. Fulkerson, Blocking and anti-blocking pairs of polyhedra, Math. Programming 1 (1971), 168-194, doi:10.1007/bf01584085.

[11] M. Funk, B. Jackson, D. Labbate and J. Sheehan, 2-factor Hamiltonian graphs, J. Comb. Theory Ser. B 87 (2003), 138-144, doi:10.1016/s0095-8956(02)00031-x.

[12] J. Hägglund and E. Steffen, Petersen-colorings and some families of snarks, Ars Math. Contemp. 7 (2014), 161-173, doi:10.26493/1855-3974.288.11a.

[13] A. Hakobyan and V. Mkrtchyan, $S_{12}$ and $P_{12}$-colorings of cubic graphs, Ars Math. Contemp. 17 (2019), 431-445, doi:10.26493/1855-3974.1758.410.

[14] F. Jaeger, Nowhere-zero flow problems, in: L. W. Beineke and R. J. Wilson (eds.), Selected Topics in Graph Theory, Volume 3, Academic Press, San Diego, CA, pp. 71-95, 1988.

[15] L. Jin and E. Steffen, Petersen cores and the oddness of cubic graphs, J. Graph Theory 84 (2017), 109-120, doi:10.1002/jgt.22014.

[16] T. Kaiser, D. Král and S. Norine, Unions of perfect matchings in cubic graphs, in: M. Klazar, J. Kratochvíl, M. Loebl, J. Matoušek, R. Thomas and P. Valtr (eds.), Topics in Discrete Mathematics, Springer, Berlin, volume 26 of Algorithms and Combinatorics, pp. 225-230, 2006, doi:10.1007/3-540-33700-8_14. 
[17] T. Kaiser and A. Raspaud, Perfect matchings with restricted intersection in cubic graphs, $E u$ ropean J. Combin. 31 (2010), 1307-1315, doi:10.1016/j.ejc.2009.11.007.

[18] D. Král', E. Máčajová, O. Pangrác, A. Raspaud, J.-S. Sereni and M. Škoviera, Projective, affine, and abelian colorings of cubic graphs, European J. Combin. 30 (2009), 53-69, doi:10.1016/j. ejc.2007.11.029.

[19] H. Lin and X. Wang, Three matching intersection property for matching covered graphs, Discrete Math. Theor. Comput. Sci. 19 (2017), \#16, doi:10.23638/dmtcs-19-3-16.

[20] L. Lovász and M. D. Plummer, Matching Theory, volume 121 of North-Holland Mathematics Studies, North-Holland, Amsterdam, 1st edition, 1986.

[21] E. Máčajová and M. Škoviera, Fano colourings of cubic graphs and the Fulkerson conjecture, Theoret. Comput. Sci. 349 (2005), 112-120, doi:10.1016/j.tcs.2005.09.034.

[22] E. Máčajová and M. Škoviera, Sparsely intersecting perfect matchings in cubic graphs, Combinatorica 34 (2014), 61-94, doi:10.1007/s00493-014-2550-4.

[23] G. Mazzuoccolo, Covering a cubic graph with perfect matchings, Discrete Math. 313 (2013), 2292-2296, doi:10.1016/j.disc.2013.06.006.

[24] G. Mazzuoccolo, New conjectures on perfect matchings in cubic graphs, Electron. Notes Discrete Math. 40 (2013), 235-238, doi:10.1016/j.endm.2013.05.042.

[25] Z. Miao, X. Wang and C.-Q. Zhang, Unique Fulkerson coloring of Petersen minor-free cubic graphs, European J. Combin. 43 (2015), 165-171, doi:10.1016/j.ejc.2014.08.021.

[26] V. V. Mkrtchyan, A remark on the Petersen coloring conjecture of Jaeger, Australas. J. Combin. 56 (2013), 145-151, https://ajc.maths.uq.edu.au/pdf/56/ajc_v56_p145. pdf.

[27] V. V. Mkrtchyan and G. N. Vardanyan, On two consequences of Berge-Fulkerson conjecture, AKCE Int. J. Graphs Comb. (2020), doi:10.1016/j.akcej.2019.03.018.

[28] J. Petersen, Die Theorie der regulären graphs, Acta Math. 15 (1891), 193-220, doi:10.1007/ bf02392606.

[29] R. Šámal, New approach to Petersen coloring, Electron. Notes Discrete Math. 38 (2011), 755 760, doi:10.1016/j.endm.2011.10.026.

[30] E. Steffen, 1-factor and cycle covers of cubic graphs, J. Graph Theory 78 (2015), 195-206, doi:10.1002/jgt.21798.

[31] Q. Zhu, W. Tang and C.-Q. Zhang, Perfect matching covering, the Berge-Fulkerson conjecture, and the Fan-Raspaud conjecture, Discrete Appl. Math. 166 (2014), 282-286, doi:10.1016/j. dam.2013.10.008. 